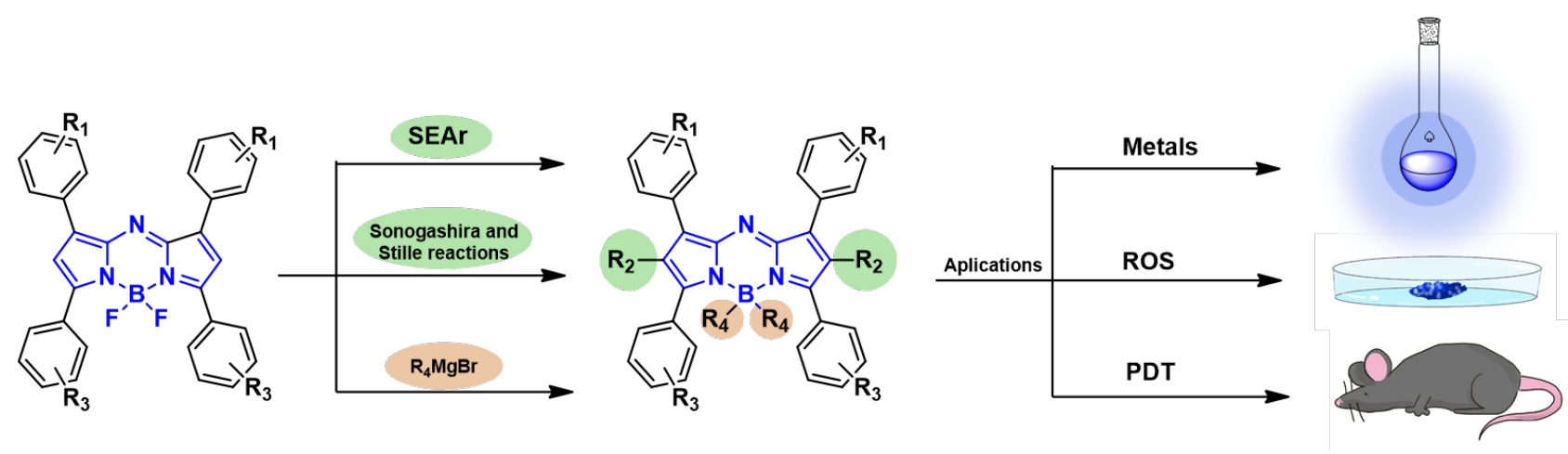

\title{
FLUOROFÓROS AZa-BODIPY: ESTRATÉGIAS DE SÍNTESE E APLICAÇÕES
}

\section{Pedro G. Spinello a, Alcindo A. Dos Santos ${ }^{\text {a,* }}$}

aDepartamento de Química Fundamental, Instituto de Química, Universidade de São Paulo, 05508-000 São Paulo - SP, Brasil.

*e-mail: pedroguillem@usp.br

\begin{abstract}
$A z a$-BODIPY FLUOROPHORES: SYNTHETIC ESTRATEGIES AND APLICATIONS

Since their discovery in 1968, the BODIPYs (4,4-difluoro-4-bora-3a, 4a-diaza-s-indacene) dyes have been attracting attention due to their wide applicability. However, these compounds have some undesirable characteristics, one being their absorption and emission below $600 \mathrm{~nm}$. To remedy this disadvantage, many researchers started to develop analogues, leading to the synthesis of a new class of fluorophores, the $a z a$-BODIPY's. This review will address the synthesis, structural modifications and applications of these interesting compounds.
\end{abstract}

Keywords: aza-BODIPY; fluorescence; bioimaging; phototherapy; fluorescence probe.

\section{Resumo}

FLUOROFOROS $A z a$-BODIPY: ESTRATÉGIAS DE SINTESE E APLICAÇÕES

Desde sua descoberta em 1968, os corantes BODIPYs (4,4-difluoro-4-bora-3a, 4a-diaza-sindacene) têm chamado a atenção devido à sua ampla aplicabilidade. No entanto, esses compostos apresentam absorção e emissão abaixo de $600 \mathrm{~nm}$. Para contornar esta pequena desvantagem, estudos recentes estão voltados para o desenvolvimento de análogos, levando à síntese de uma nova classe de fluoroforos, os aza-BODIPY'. Esta revisão abordará a síntese, modificações estruturais e aplicações desses compostos.

Palavras-chave: aza-BODIPY; fluorescência; bioimagem; fototerapia; sonda de fluorescência. 


\section{Fluoróforos}

Compostos orgânicos fluorescentes são substâncias que têm a capacidade de emitir luz ao absorver radiação eletromagnética ${ }^{1}$. Tais espécies se constituem em ferramentas poderosas, que estão à disposição de pesquisadores que investigam eventos e processos químicos, físicos e biológicos ${ }^{2}$. Nos últimos anos, tem havido grandes esforços no design e síntese de fluoróforos com estruturas moleculares inéditas e propriedades fotofísicas-chave para as mais diversas aplicações ${ }^{3}$.

Alguns cromóforos têm sido utilizados como sensores moleculares ${ }^{3}$, como marcadores fluorescentes ${ }^{4}$, como diodos orgânicos emissores de luz (OLEDs) ${ }^{5}$ e como sensibilizantes de células fotovoltaicas ${ }^{6}$. Além disso, o interesse nestes materiais vem se intensificando, devido aos seus relevantes usos em sistemas biológicos e aplicações para imageamento em medicina ${ }^{7}$.

Apesar do extenso acervo de fluoróforos que vêm sendo utilizados e estudados, a família dos BODIPY's (boron dipirromethene) tem chamado atenção devido ao acesso sintético, facilidade de funcionalização, alta estabilidade química, versatilidade e boas propriedades fotofísicas $^{8-10}$.

\section{Histórico}

O núcleo BODIPY (4,4-difluoro-4-bora-3a,4a-diaza-s-indaceno), apresentado na figura 1 (A), foi sintetizado pela primeira vez por Treibs e Kreuzer em $1968^{11}$. Desde então, tem sido vastamente explorado e aplicado em diversas áreas do conhecimento, porém apresentam algumas características fotofísicas que limitam suas aplicações ${ }^{8}$. Este fato estimulou investigações de sistemas similares, que não apresentassem tais limitações.

Uma das principais limitações dos BODIPY's é a faixa de absorção e emissão, que está no ultravioleta-visível (UV-Vis), comprimento de onda em que ocorre a absorção e/ou espalhamento de luz em biomateriais e consequente limitação de uso. Para muitas aplicações mais sofisticadas, especialmente em biomedicina e bioquímica, é necessário que o fluoróforo, absorva no infravermelho próximo (IVP) ${ }^{12}$ e com isso, grandes avanços foram e vem sendo atingidos com derivados aza-BODIPY (figura 1B, Os BODIPY's são caracterizados por sais internos e por efeitos práticos, as cargas não serão apresentadas nas próximas estruturas).

A)

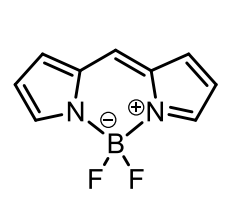

B)

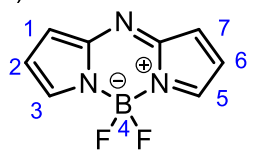

Figura 1. Núcleos fundamentais de um BODIPY e de um aza-BODIPY.

O primeiro registro de reação envolvendo um aza-dipirrometeno (1) foi descrito em 1943 por Rogers ${ }^{13}$, porém esta classe recebeu pouca atenção e somente em 1993 Boyer demonstrou que sua complexação com o trifluoreto de boro ${ }^{14}$. O complexo di-fluorborado 2 
apresentou características fluorescentes interessantes e destacadamente relevantes, em comparação com seu precursor; contudo a rota sintética empregada por Rogers apresentava condições drásticas e baixo rendimento, o que motivou a realização de novos estudos.
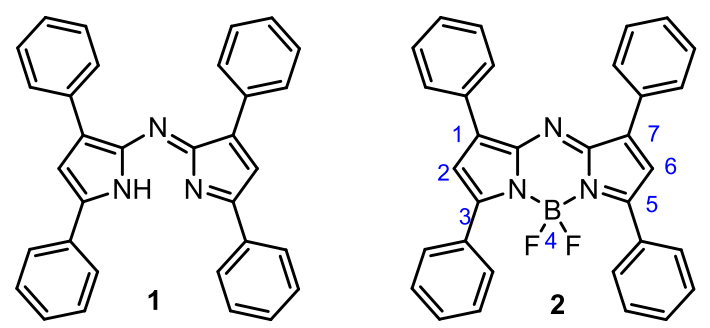

Figura 2. Representação estrutural dos primeiros aza-dipirrometeno e aza-BODIPY reportados em literatura.

\section{Principais rotas sintéticas}

\section{DESIGN ESTRUTURAL}

Devido a necessidade de rotas sintéticas em condições amenas, com maiores rendimentos e operacionalmente mais fáceis para a síntese do núcleo $a z a$-BODIPY, diversos estudos metodológicos foram realizados e entre eles, os três principais foram os de O'Shea, Carreira e Lukyanets, como apresentado na figura 3.

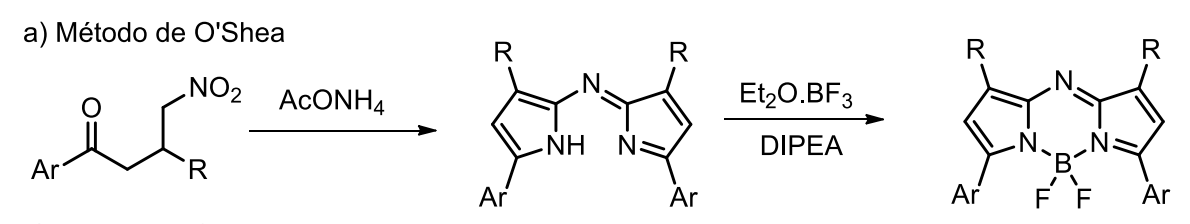

b) Método de Carreira

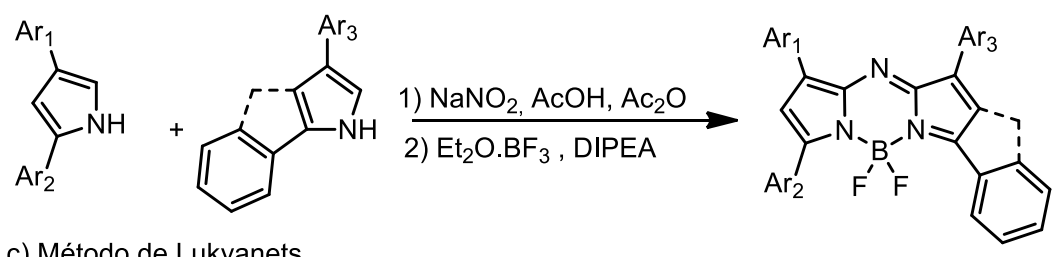

c) Método de Lukyanets

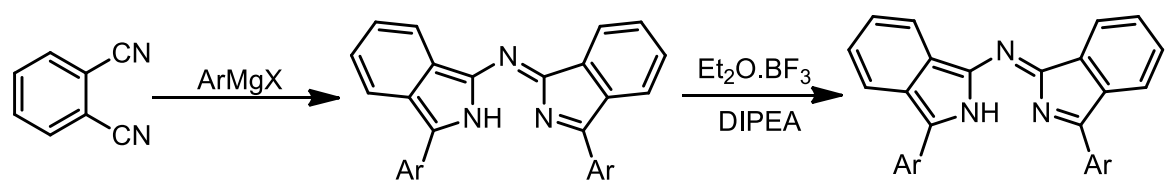

Figura 3. Principais rotas sintéticas para a construção do núcleo aza-BODIPY

Em um primeiro momento, O'Shea investigou a síntese dos produtos simétricos e assimétricos ${ }^{15}$, partindo da ciclização dos adutos obtidos da adição 1,4- do nitrometano à chalconas, seguido de boro-complexação. As condições reacionais deste método são amenas e os rendimentos globais variam de 25 a $60 \%$. 
O método descrito por Carreira permite explorar a extensão da conjugação de compostos $a z a$-BODIPY e se fundamenta na ciclização direta de pirróis substituídos, seguida da complexação ${ }^{16}$. Por este procedimento se pode preparar compostos simétricos e assimétricos, em rendimentos tipicamente maiores que os da metodologia comentada anteriormente, ficando na faixa de 40 a $90 \%$.

Em estudos mais recentes, Lukyanets demonstrou uma metodologia que permitiu preparar compostos simétricos ao submeter a ftalonitrila à reação com halogeneto de arilmagnésio ${ }^{17}$. Esta condição reacional apresenta rendimentos aproximadamente de $20 \%$, inferiores aos exemplos reportados anteriormente.

Estas são as três principais e mais abrangentes rotas sintéticas de $a z a$-BODIPY's; nos núcleos já sintetizados, várias modificações estruturais podem ser realizadas à fim de modular as características e propriedades desejáveis nos compostos-alvo ${ }^{18}$. Dentre estas características as mais realizadas são modificações para melhorar características fotofísicas $^{16,19,20} \mathrm{e}$ aumentar hidrofilicidade/biocompatibilidade ${ }^{21-24}$.

\section{Modificações estruturais}

Estruturalmente, os aza-BODIPY's são versáteis e admitem funcionalizações/modificações estratégicas, a partir de núcleos fundamentais mais gerais, permitindo síntese de famílias de compostos. Intrinsecamente, a presença de anéis aromáticos nas posições 1, 3, 5 e 7 lhes conferem maior estabilidade e por consequência, em compostos que contenham anéis nestas posições, pós-funcionalizações das posições 2,4 e 6 estão entre as modificações mais comumente reportadas em literatura.

O primeiro relato da substituição do núcleo aza-BODIPY foi de halogenação, onde foi realizada reação de bromação ${ }^{25}$ nas posições 2 e 6 do $a z a$-dipirrometeno (1) utilizando $\mathrm{Br}_{2}$. Para a formação deste produto as condições reacionais empregadas foram amenas, levando ao núcleo dihalogenado (3) em rendimentos elevados (Figura 4). Em seguida, observou-se que com substituintes ativantes, também é possível realizar a halogenação com o núcleo já complexado utilizando de $N$-iodosuccinimida (NIS) ou de $N$-bromosuccinimida (NBS) ${ }^{26,27}$.

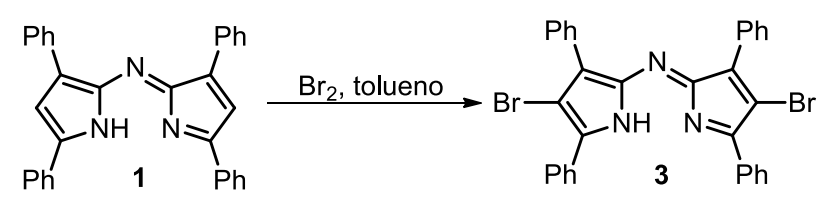

Figura 4. Estratégia de halogenação do composto 1

Em seguida, Jiao em $2009^{28}$ foi pioneiro na monosubstituição de um grupamento aldeído no núcleo, tornando o núcleo um bloco de construção estratégico. A reação de formilação ocorreu via reação de Vilsmeier-Haack, produzindo o correspondente $a z a$-BODIPY monosubstituído (4) em alto rendimento (Figura 5). O produto diformilado não foi observado devido ao fato da primeira inserção da carbonila, que implicou em desativação, prevenindo o segundo ataque nucleofílico. 


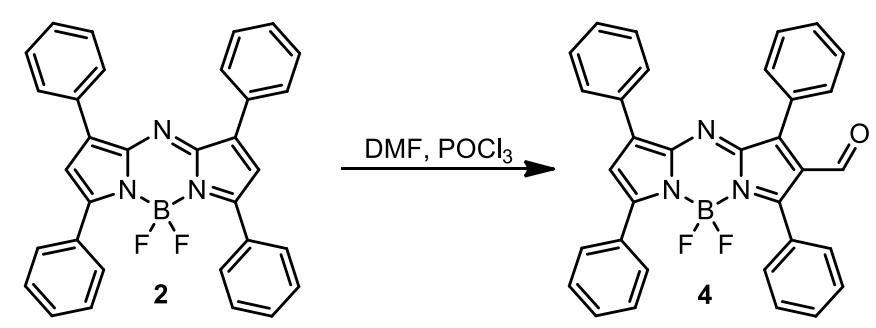

Figura 5. Condição de formilação do núcleo aza-BODIPY

O composto monoformilado que tem um grupamento muito eletrofílico, é submetido a reações de Wittig utilizando uma ilida de fósforo estabilizada, dando origem aos correspondentes produtos $\alpha, \beta$-insaturados em rendimentos da ordem de 50 a $90 \%{ }^{29,30}$. Além disso, também é possível a reação do aldeído com grupos nucleofílicos, para formação de compostos para aplicações variadas ${ }^{31,32}$.

Os núcleos halogenados também podem ser utilizados como blocos de construção para reações posteriores, principalmente para reações de acoplamento catalisadas por paládio. Esta reação foi reportada pela primeira vez com núcleos $a z a$-BODIPY, em 2011 por Moritz e colaboradores, em que utilizaram o acoplamento de Stille como estratégia de acoplamento de um composto dibromado com um tiofeno silanizado que levou ao 2,6-tiofeno azaBODIPY em bom rendimento (figura 6) ${ }^{33}$.
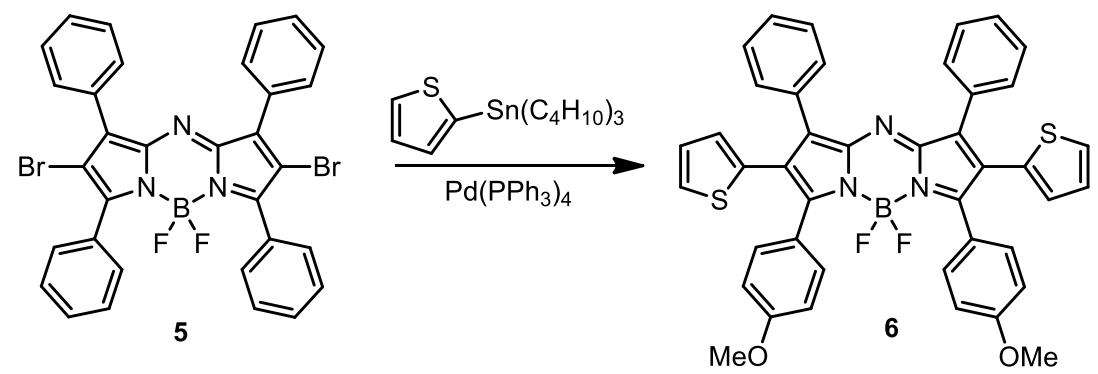

Figura 6. Reação de acoplamento de Stille para formação do 2,6-tiofeno aza-BODIPY

Em um estudo recente um acoplamento de Sonogashira, foi reportado por Pascal e coautores, como estratégia de preparação de BODIPY's di-alquinicos $(\mathbf{8})$, em rendimentos elevados. Estes compostos foram investigados para a aplicação como limitadores de potência óptica (Figura 7) ${ }^{34}$. 

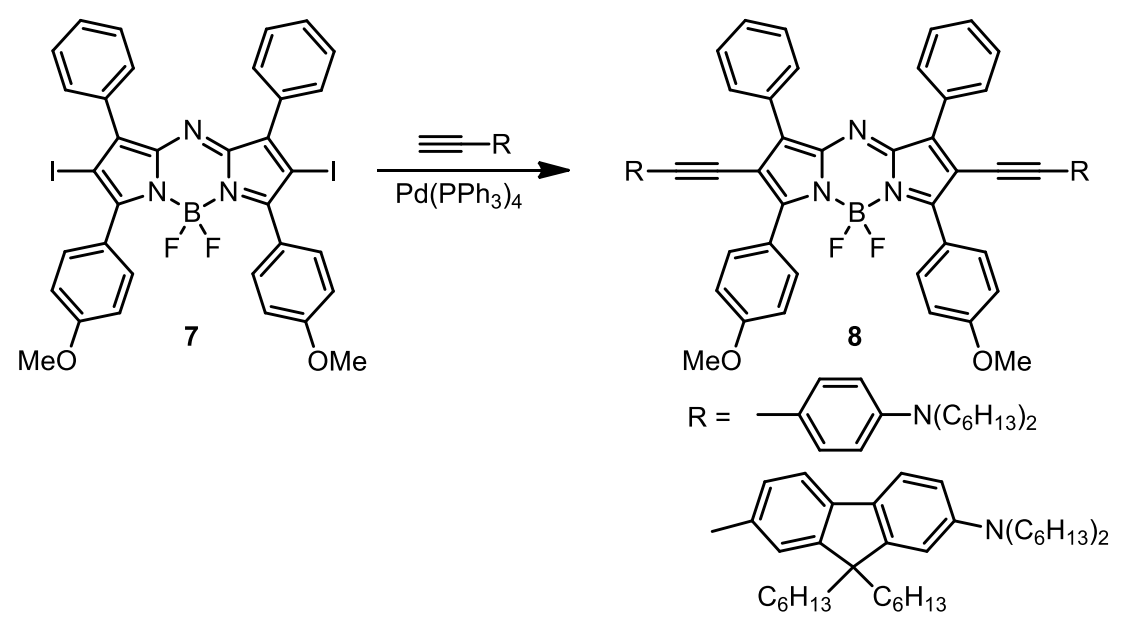

Figura 7. Exemplo de reação de acoplamento de aza-iodo-BODIPY's com alquinos terminais, catalisada por paládio

Como já comentado, além das posições 2 e 6 serem passíveis de funcionalizações, também é possível realizar modificações na posição 4. A funcionalização do átomo de boro é uma estratégia amplamente investigada nos corantes de núcleo BODIPY ${ }^{35}$, porém menos comuns em derivados $a z a$-BODIPY. A substituição dos átomos de flúor foi reportada por O'Shea ${ }^{36}$, onde realizou a reação do $a z a$-BODIPY com metóxido de sódio, dando origem ao composto 9 (Figura 8).
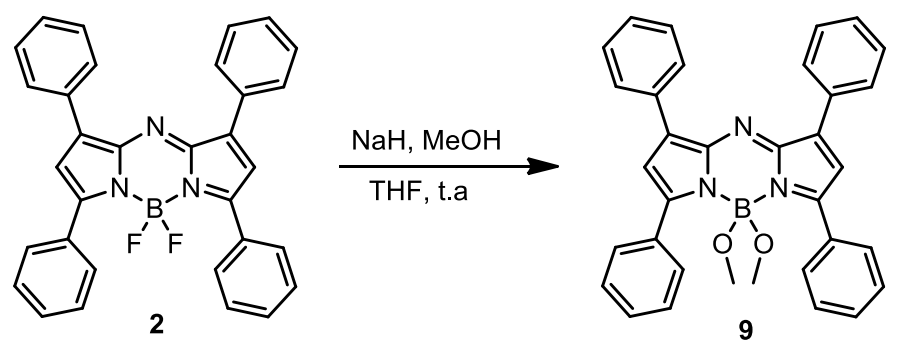

Figura 8. Primeira reação de substituição dos átomos de flúor de um aza-BODIPY

$\mathrm{O}$ ataque nucleofílico de reagentes de Grignard são as principais estratégias empregadas atualmente para a substituição dos átomos de flúor da posição 4, como representado na figura $9^{35}$. Recentemente foram reportadas ${ }^{22,37-39}$ sínteses de $a z a$-BODIPY's com este padrão de substituições, sendo que alguns dos produtos gerados tiveram sua hidrofilicidade aumentada, permitindo aplicações em sistemas biológicos in-vitro e in-vivo.

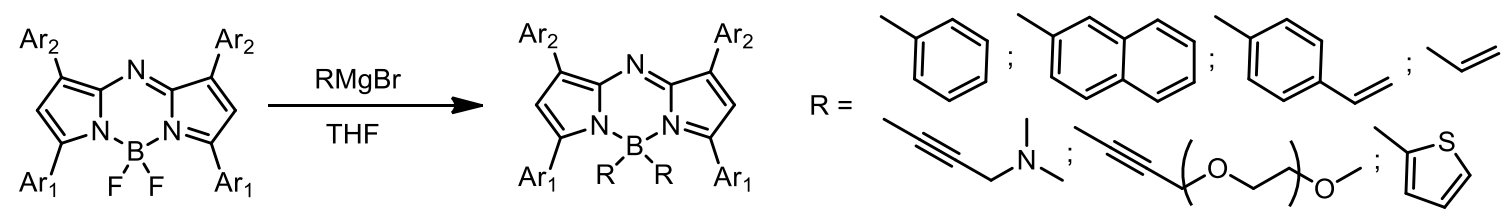


Figura 9. Principal estratégia para substituição dos átomos de flúor

\section{APLICAÇÕES}

\section{Sondas fluorescentes}

A maioria dos eventos estudados nas ciências química, biológica e física ocorrem em escalas muito menores dos que grande parte de outros eventos cotidianos com os quais a maioria das pessoas está acostumada a presenciar. Algumas informações sobre esses eventos podem ser convenientemente transmitidas por meio de sinais de luz, que são emitidos por dispositivos moleculares modelados, idealmente para a deteç̧ão de certo analito. Entre estes dispositivos estão as sondas fluorescentes ${ }^{3}$.

Este recurso analítico tem ganhado cada vez mais aplicações e versatilidade devido à alta sensitividade, baixo tempo de resposta, alta resolução e seletividade, com baixa ou mínima invasividade $^{40}$. Os BODIPY's e $a z a$-BODIPY's encontram grandes e amplas aplicações como sensores fluorescentes devido a suas excelentes propriedades fotofísicas, sendo possível a modulação destas propriedades em função do perfil estrutural requerido para a aplicação pretendida.

\section{Detecção de espécies reativas de oxigênio}

$\mathrm{O}$ oxigênio tem uma estrutura molecular única e além de muito abundante em organismos vivos, cumprindo papéis vitais, é precursor ou componente de entidades químicas responsáveis por estresse oxidativo, que também cumpre papéis benéficos e deletérios. Ao aceitar elétrons, em processos do metabolismo aeróbio, da origem às espécies reativas de oxigênio (ERO's), mediadoras dos processos de estresse oxidativo ${ }^{41}$. Cabem a estas espécies várias funções vitais, entre as quais a sinalização celular, indução de necrose, supressão ou indução de expressão de genes entre outras ${ }^{42}$. Contudo, o desequilíbrio na produção das ERO's e das defesas antioxidantes que protegem as células pode implicar em danos, causando assim inúmeras doenças ou desordens como câncer, asma e problemas neurodegenerativos como Alzheimer e Parkinson ${ }^{41-44}$. Devido as patologias associadas as ERO's, sua detecção, quantificação e especiação são de fundamental importância para a compreensão, diagnostico e controle/tratamento dos eventos aos quais estão relacionadas. As sondas fluorescentes estão entre as principais ferramentas analíticas rotineiramente desenvolvidas, customizadas e sintetizadas para a medição das ERO's.

Uma das primeiras divulgações cientificas de $a z a$-BODIPY para detecção de ERO's foi realizada por Jiang e colaboradores em $2016^{45}$. Neste trabalho, os autores mostraram que a função "ácido borônico" nos grupamentos arilas, (composto 10) das posições 3 e 7, reagiam de maneira seletiva com $\mathrm{H}_{2} \mathrm{O}_{2}$ (Figura 10), convertendo o ácido borônico nos respectivos fenóis (composto 11). Simultaneamente a esta transformação, ocorre a diminuição de emissão fluorescência em $681 \mathrm{~nm}$ e aumento em $724 \mathrm{~nm}$ evidenciando a presença de peróxido de hidrogênio no meio. Também foram realizados estudos in vitro a fím de detectar estas espécies utilizando-se de células hela. 


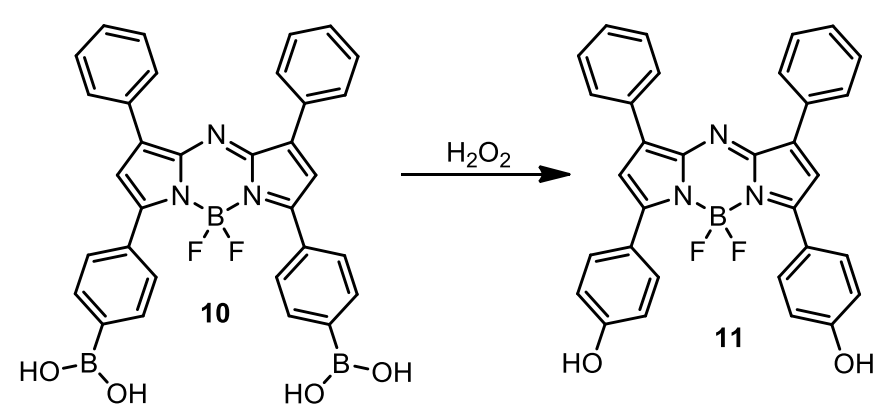

Figura 10. Reação seletiva da sonda na detecção de $\mathrm{H}_{2} \mathrm{O}_{2}$

Outros derivados $a z a$-BODIPY funcionalizados com grupamentos derivados do ácido borônico ${ }^{46}$ e derivados de hidrazonas ${ }^{47}$ foram utilizados para detecção de ERO's. Neste ano (2021), Gao e co-autores ${ }^{48}$ reportaram a síntese e aplicação de um sensor seletivo para íons hipoclorito $\left(\mathrm{ClO}^{-}\right)$, representado pelo composto 12. O evento de oxidação promovido por íons hipoclorito foi detectado pela oxidação seletiva dos átomos de telúrio, que atuaram como "chaves liga/desliga" do dispositivo fluorescente, formando o produto 13. Neste estudo, o fenômeno de fluorescência foi mediado pelos átomos de telúrio graças ao processo de transferência eletrônica fotoinduzida (PET), trazido pelo efeito do átomo pesado do telúrio ${ }^{49}$. Neste trabalho, foram realizados estudos in vitro com células RAW264.7 em que íons hipoclorito cumprem papel fisiológico relevante (Figura 11).

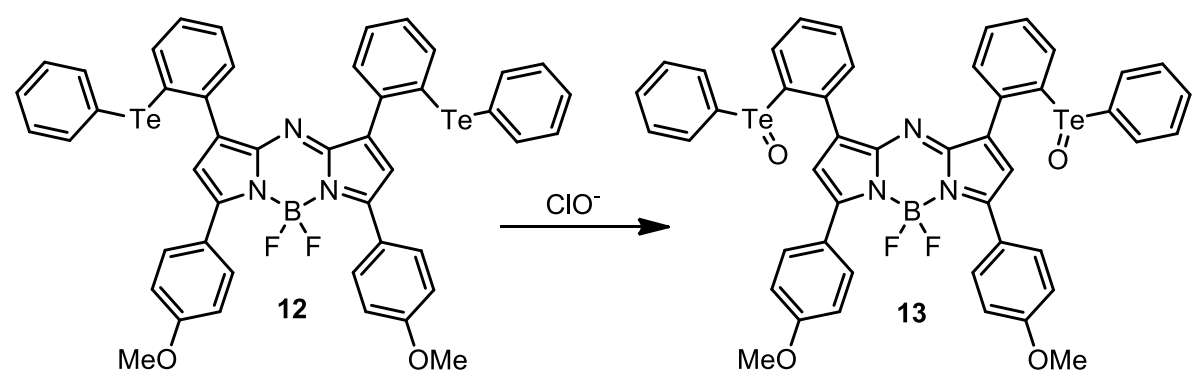

Figura 11. Reação de oxidação dos átomos de telúrio do aza-BODIPY promovida seletivamente por ions hipoclorito, acompanhado de aumento de fluorescência

\section{Detecção de metais}

Outra aplicação para as quais os sensores fluorescentes tem ganhado grande aplicação é a de detecção de íons metálicos, em diferentes matrizes, incluindo o de amostras ambientais e biológicas. O mercúrio está entre os elementos de maior toxicidade para organismos superiores, em todos os seus estados de oxidação ${ }^{50}$.

Akkaya e colaboradores reportaram a detecção de metais promovida por aza-BODIPY's, substituídos nas posições 1 e 7 por grupamentos 2-piridinicos, que desempenharam papel quelante de íons metálicos ${ }^{51}$. A sonda apresentou boa seletividade para íns $\mathrm{Hg}^{2+}$, evidenciado pelo deslocamento de emissão de fluorescência, de 682 para $719 \mathrm{~nm}$ (Figura 
12). Sondas contendo grupamentos 2-piridinil ${ }^{52}$ e 2-tiofeno ${ }^{53}$ também foram investigadas para tais aplicações.

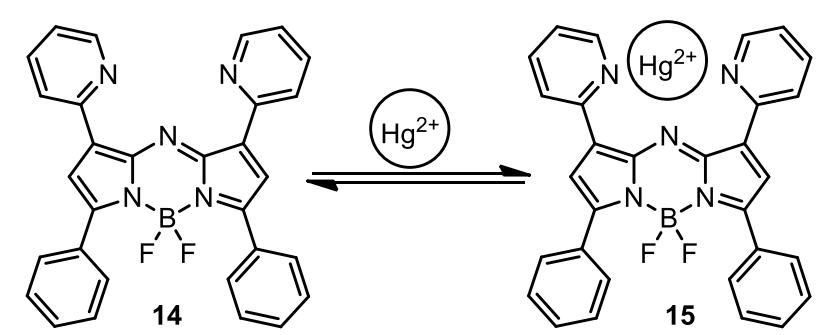

Figura 12. Representação da estrutura formada da interação do composto 14 com ions $\mathrm{Hg}^{2+}$

O Cobre é um elemento-traço, presente em organismos biológicos com participação em processos celulares importantes ${ }^{54,55}$. Contudo em altas concentrações pode ser tóxico e o aumento de sua utilização em vários segmentos, em especial agricultura, provoca contaminação ambiental que pode acarretar em doenças neurodegenerativas ${ }^{56}$ e por este motivo, métodos robustos de sua detecção são importantes.

Em estudos recentes, Sekar e co-autores reportaram a síntese de um aza-BODIPY substituído com uma porção 3 -etóxi- $N, N$-dietilanilina nos anéis aromáticos superiores (16) $^{57}$, capaz de detectar quantitativa- e seletivamente, íons cobre (Figura 13). O composto 16 apresentou emissão apenas residual em $749 \mathrm{~nm}$ e na presença de $\mathrm{Cu}^{2+}$ passou a ter forte fluorescência em $700 \mathrm{~nm}$.

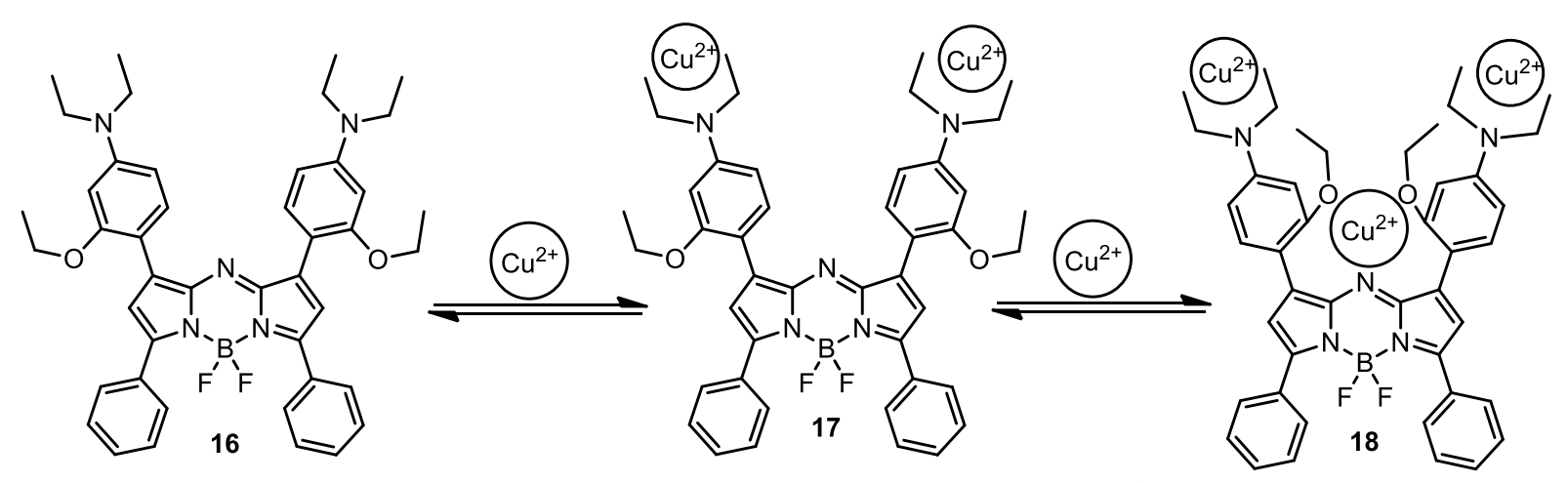

Figura 13. Esquema de reação entre o aza-BODIPY 16 e ions $\mathrm{Cu}^{2+}$

\section{Terapia fotodinâmica}

Terapia fotodinâmica (PDT) consiste na habilidade de um composto ou sistema, de no estado excitado, reagir com oxigênio para formar oxigênio singleto $\left({ }^{1} \mathrm{O}_{2}\right)$. A espécie resultante pode reagir com as moléculas presentes na célula causando estresse oxidativo, ocasionando, consequentemente, morte celular ${ }^{21,58}$. Derivados aza-BODIPY's, além de 
poderem atuar como poderosos sensores de analitos variados, podem agir como fotossensibilizantes em processos relacionados a PDT ${ }^{12}$.

A principal estratégia para aumentar o cruzamento intersistemas singleto-tripleto (ISC), em compostos orgânicos capazes de desempenhar este papel, é a inserção de átomos pesados como iodo, bromo, cloro, selênio ${ }^{59}$, enxofre ${ }^{60}$, rutênio, irídio. Porém, entre estes exemplos, o iodo apresenta os melhores resultados e também estes efeitos são maximizados quando ligados diretamente ao núcleo fluorescente ${ }^{61}$.

Alguns derivados $a z a$-BODIPY's vêm sendo preparados $\mathrm{e}$ destinados à terapia fotodinâmica ${ }^{54,58}$. Recentemente, Huang e colaboradores relataram a utilização do composto diiodado no tratamento in vivo de tumor em ratos (Figura 14) ${ }^{62}$. Para gerar oxigênio singlete, radiação no comprimento de onda de $660 \mathrm{~nm}$ foi incidida na área do tumor, previamente tratado com o fotossensibilizante 19. O desenvolvimento do tumor foi analisado comparativamente (durante 17 dias) em presença de 19 na presença e ausência de luz. O tratamento em presença de 19, que recebeu irradiação levou à morte celular (no tecido tumoral), confirmando a produção de oxigênio singlete e sucesso da aplicação do composto para PDT.

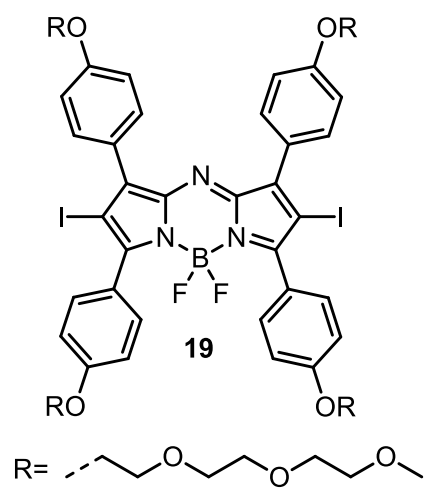

Figura 14. Representação estrutural do fotossensibilizante utilizado nos ensaios in vivo

\section{CONCLUSÃO}

Após o trabalho pioneiro de O'Shea e colaboradores, publicado em $2002^{15}$, compostos $a z a$ BODIPY's passaram a atrair significativamente a atenção da comunidade cientifica com aplicações que vão da detecção de analitos de interesse ambiental como íons metálicos ao tratamento de câncer in vivo. Do ponto de vista prático, a utilização de $a z a$-BODIPY's é bastante atrativa, pois em geral suas sínteses são relativamente fáceis e robustas, com possibilidade de diversidade estrutural e modulação de propriedades fotofísicas e excelentes fotoestabilidade, além de apresentarem faixas de comprimentos de onda de absorção e emissão na região do IVP, compatíveis com sistemas biológicos para aplicações in vivo. Nos últimos cinco anos, a pesquisa relacionada a síntese de novos núcleos $a z a$-BODIPY e de novas modificações estruturais tiveram um rápido desenvolvimento devido às aplicações tecnológicas que estes compostos vêm ganhando. Apesar disso, as aplicações de maior 
relevância e importância, que se referem ao campo da medicina ainda são muito recentes, contudo, promissoras e com uma janela de oportunidades não só instigantes do ponto de vista cientifico, como também decisivas do ponto de vista do impacto social e de saúde pública que podem trazer.

\section{REFERÊNCIAS}

1. Jameson, D. M. Jameson, David M-Introduction to Fluorescence-CRC Press (2014); 2014.

2. Bismillah, A. N.; Aprahamian, I. ;Chem. Soc. Rev. 2021, 50, 5631.

3. Demchenko, A. P. Introduction to Fluorescence Sensing; 2015.

4. Sameiro, M.; Gonçalves, T. ;Chem. Rev. 2009, 109, 190.

5. Chou, P. T.; Chi, Y. ;Chem. - A Eur. J. 2007, 13, 380.

6. Mishra, A.; Fischer, M. K. R.; Büuerle, P. ;Angew. Chemie - Int. Ed. 2009, 48, 2474.

7. Grimm, J. B.; Tkachuk, A. N.; Xie, L.; Choi, H.; Mohar, B.; Falco, N.; Schaefer, K.; Patel, R.; Zheng, Q.; Liu, Z.; Lippincott-Schwartz, J.; Brown, T. A.; Lavis, L. D. ;Nat. Methods 2020, 17, 815 .

8. Loudet, A.; Burgess, K. BODIPY dyes and their derivatives: Syntheses and spectroscopic properties. Chem. Rev. 2007, 107, 4891-4932.

9. Kowada, T.; Maeda, H.; Kikuchi, K. ;Chem. Soc. Rev. 2015, 44, 4953.

10. Ulrich, G.; Ziessel, R.; Harriman, A. ;Angew. Chemie - Int. Ed. 2008, 47, 1184.

11. Treibs, A.; Kreuzer, F. -H ;Justus Liebigs Ann. Chem. 1968, 718, 208.

12. Kobayashi, H.; Ogawa, M.; Alford, R.; Choyke, P. L.; Urano, Y. ;Chem. Rev. 2010, 110, 2620.

13. Rogers, M. A. T. ; J. Chem. Soc. 1943, 590.

14. Sathyamoorthi, G.; Soong, M.; Ross, T. W.; Boyer, J. H. 1993, 4, 603.

15. Killoran, J.; Allen, L.; Gallagher, J. F.; Gallagher, W. M.; O’Shea, D. F. ;Chem. Commun. 2002, 317, 1862.

16. Zhao, W.; Carreira, E. M. ;Chem. - A Eur. J. 2006, 12, 7254.

17. Donyagina, V. F.; Shimizu, S.; Kobayashi, N.; Lukyanets, E. A. ;Tetrahedron Lett. 2008, 49, 6152.

18. Shamova, L. I.; Zatsikha, Y. V.; Nemykin, V. N. Synthesis pathways for the preparation of the BODIPY analogues: aza-BODIPYs, BOPHYs and some other pyrrole-based acyclic chromophores. Dalt. Trans. 2021, 50, 1569-1593.

19. Wang, Y.; Zhang, D.; Xiong, K.; Shang, R.; Jiang, X. D. ;Chinese Chem. Lett. 2021.

20. Daly, H. C.; Sampedro, G.; Bon, C.; Wu, D.; Ismail, G.; Cahill, R. A.; O’Shea, D. F. ;Eur. J. Med. Chem. 2017, 135, 392.

21. Kalot, G.; Godard, A.; Busser, B.; Pliquett, J.; Broekgaarden, M.; Motto-Ros, V.; Wegner, K. D.; Resch-Genger, U.; Köster, U.; Denat, F.; Coll, J. L.; Bodio, E.; Goze, C.; Sancey, L. ;Cells 2020, 9, 2.

22. Kalot, G.; Godard, A.; Busser, B.; Pliquett, J.; Broekgaarden, M.; Motto-Ros, V.; 
Wegner, K. D.; Resch-Genger, U.; Köster, U.; Denat, F.; Coll, J. L.; Bodio, E.; Goze, C.; Sancey, L. ;Cells 2020, 9.

23. Kamkaew, A.; Burgess, K. ;Chem. Commun. 2015, 51, 10664.

24. Wu, D.; Cheung, S.; Daly, R.; Burke, H.; Scanlan, E. M.; O’Shea, D. F. ;European J. Org. Chem. 2014, 2014, 6841.

25. Gorman, A.; Killoran, J.; O’Shea, C.; Kenna, T.; Gallagher, W. M.; O’Shea, D. F. ;J. Am. Chem. Soc. 2004, 126, 10619.

26. Yu, Z.; Zhou, J.; Ji, X.; Lin, G.; Xu, S.; Dong, X.; Zhao, W. ;J. Med. Chem. 2020, 63, 9950.

27. Karatay, A.; Miser, M. C.; Cui, X.; Küçüköz, B.; Yilmaz, H.; Sevinç, G.; Akhüseyin, E.; Wu, X.; Hayvali, M.; Yaglioglu, H. G.; Zhao, J.; Elmali, A. ;Dye. Pigment. 2015, $122,286$.

28. Jiao, L.; Yu, C.; Li, J.; Wang, Z.; Wu, M.; Hao, E. ;J. Org. Chem. 2009, 74, 7525.

29. Koch, A.; Kumar, S.; Ravikanth, M. ;Tetrahedron 2017, 73, 1459.

30. Wang, J.; Wu, Y.; Sheng, W.; Yu, C.; Wei, Y.; Hao, E.; Jiao, L. ;ACS Omega 2017, 2 , 2568.

31. Kumar, S.; Gobeze, H. B.; Chatterjee, T.; Ravikanth, M. 1.

32. Gao, Y.; Pan, Y.; Chi, Y.; He, Y.; Chen, H.; Nemykin, V. N. ;Spectrochim. Acta Part A Mol. Biomol. Spectrosc. 2019, 206, 190.

33. Gresser, R.; Hartmann, H.; Wrackmeyer, M.; Leo, K.; Riede, M. ;Tetrahedron 2011, 67, 7148.

34. Pascal, S.; Bellier, Q.; David, S.; Bouit, P. A.; Chi, S. H.; Makarov, N. S.; Le Guennic, B.; Chibani, S.; Berginc, G.; Feneyrou, P.; Jacquemin, D.; Perry, J. W.; Maury, O.; Andraud, C. ;J. Phys. Chem. C 2019.

35. Bodio, E.; Goze, C. ;Dye. Pigment. 2019, 160, 700.

36. Palma, A.; Tasior, M.; Frimannsson, D. O.; Vu, T. T.; Méallet-Renault, R.; O'Shea, D. F. ;Org. Lett. 2009, 11, 3638.

37. Florès, O.; Pliquett, J.; Abad Galan, L.; Lescure, R.; Denat, F.; Maury, O.; Pallier, A.; Bellaye, P. S.; Collin, B.; Même, S.; Bonnet, C. S.; Bodio, E.; Goze, C. ;Inorg. Chem. 2020, 59, 1306.

38. Pliquett, J.; Dubois, A.; Racoeur, C.; Mabrouk, N.; Amor, S.; Lescure, R.; Bettaïeb, A.; Collin, B.; Bernhard, C.; Denat, F.; Bellaye, P. S.; Paul, C.; Bodio, E.; Goze, C. ;Bioconjug. Chem. 2019, 30, 1061.

39. Diaz-Rodriguez, R. M.; Burke, L.; Robertson, K. N.; Thompson, A. ;Org. Biomol. Chem. 2020, 18, 2139.

40. De Silva, A. P.; Gunaratne, H. Q. N.; Gunnlaugsson, T.; Huxley, A. J. M.; McCoy, C. P.; Rademacher, J. T.; Rice, T. E. ;Chem. Rev. 1997, 97, 1515.

41. Auten, R. L.; Davis, J. M. ;Pediatr. Res. 2009, 66, 121.

42. Hancock, J. T.; Desikan, R.; Neill, S. J. ;Biochem. Soc. Trans. 2001, 29, 345.

43. Deshmukh, P. P.; Navalkar, A.; Maji, S. K.; Manjare, S. T. ;Sensors Actuators, $B$ Chem. 2019, 281, 8 . 
44. Malankar, G. S.; Sakunthala, A.; Navalkar, A.; Maji, S. K.; Raju, S.; Manjare, S. T. ;Anal. Chim. Acta 2021, 1150, 338205.

45. Xu, J.; Zhai, J.; Xu, Y.; Zhu, J.; Qin, Y.; Jiang, D. ;Analyst 2016, 141, 2380.

46. Mao, W.; Zhu, M.; Yan, C.; Ma, Y.; Guo, Z.; Zhu, W. ;ACS Appl. Bio Mater. 2020, 3, 45.

47. Gao, Y.; Pan, Y.; He, Y.; Chen, H.; Nemykin, V. N. ;Sensors Actuators, B Chem. 2018, 269, 151.

48. Shi, W. J.; Feng, L. X.; Wang, X.; Huang, Y.; Wei, Y. F.; Huang, Y. Y.; Ma, H. J.; Wang, W.; Xiang, M.; Gao, L. ;Talanta 2021, 233, 122581.

49. Daly, B.; Ling, J.; De Silva, A. P. ;Chem. Soc. Rev. 2015, 44, 4203.

50. Nolan, E. M.; Lippard, S. J. ;Chem. Rev. 2008, 108, 3443.

51. Coskun, A.; Yilmaz, M. D.; Akkaya, E. U. ;Org. Lett. 2007, 9, 607.

52. Jiang, X. D.; Zhao, J.; Li, Q.; Sun, C. L.; Guan, J.; Sun, G. T.; Xiao, L. J. ;Dye. Pigment. 2016, 125, 136.

53. Liu, S.; Shi, Z.; Xu, W.; Yang, H.; Xi, N.; Liu, X.; Zhao, Q.; Huang, W. ;Dye. Pigment. 2014, 103, 145.

54. Shi, Z.; Han, X.; Hu, W.; Bai, H.; Peng, B.; Ji, L.; Fan, Q.; Li, L.; Huang, W. Bioapplications of small molecule Aza-BODIPY: From rational structural design to: From vivo investigations. Chem. Soc. Rev. 2020, 49, 7533-7567.

55. Ciesienski, K. L.; Hyman, L. M.; Derisavifard, S.; Franz, K. J. ;Inorg. Chem. 2010, 49, 6808.

56. Saleem, M.; Rafiq, M.; Hanif, M.; Shaheen, M. A.; Seo, S. Y. A Brief Review on Fluorescent Copper Sensor Based on Conjugated Organic Dyes; Springer US, 2018; Vol. 28.

57. Gawale, Y.; Mangalath, S.; Adarsh, N.; Joseph, J.; Ramaiah, D.; Sekar, N. ;Dye. Pigment. 2019, 171, 107684.

58. Bodio, E.; Denat, F.; Goze, C. In Porphyrin Science By Women (In 3 Volumes); World Scientific Publishing Co., 2020; pp. 116-140.

59. Hoover, G. C.; Seferos, D. S. ;Chem. Sci. 2019, 10, 9182.

60. Lee, D. R.; Lee, K. H.; Shao, W.; Kim, C. L.; Kim, J.; Lee, J. Y. ;Chem. Mater. 2020, $32,2583$.

61. Ortiz, M. J.; Agarrabeitia, A. R.; Duran-Sampedro, G.; Bañuelos Prieto, J.; Lopez, T. A.; Massad, W. A.; Montejano, H. A.; García, N. A.; Lopez Arbeloa, I. ;Tetrahedron 2012, 68, 1153.

62. Miao, X.; Hu, W.; He, T.; Tao, H.; Wang, Q.; Chen, R.; Jin, L.; Zhao, H.; Lu, X.; Fan, Q.; Huang, W. ;Chem. Sci. 2019, 10, 3096. 